\title{
BOOK RECOMMENDATION SYSTEM USING OPINION MINING TECHNIQUE
}

\author{
Pranav Bhure ${ }^{1}$, Navinkumar Adhe $^{2}$ \\ ${ }^{1}$ Computer Science/ Vishwakarma Institute of Information Technology/ SavitriBai Phule Pune Unversity, Pune, India \\ ${ }^{2}$ Computer Science/ Vishwakarma Institute of Information Technology/ SavitriBai Phule Pune Unversity, Pune, India
}

\begin{abstract}
The purpose of this project is to create and deploy a book recommendation system that will help people to recommend books. Our project is the online system that helps people to get reviews about the books and give recommendations to them. Online recommendation system will also allow the users to give feedback comments that will be analyzed by opinion mining technique so as to imply the true nature of the comment .i .e whether the comment is positive, negative or a neutral one. People then searching for a particular book will be displayed with the top 10(approx.) books on that particular subject based on the reviews and feedbacks given by the earlier people who read the same book.
\end{abstract}

Keywords: - Books, Recommendation, User reviews, Opinion mining, Feedback

$* * *$

\section{INTRODUCTION}

With the changing trends in technologies, and fast growth in Internet, daily life of an individual has also changed at a very fast pace. The impacts of these technologies are so diverse that it has affected almost every aspects of the life. Internet is very frequently used by people and online shopping is preferred these days. For academician, faculties, librarians and students, purchasing the desired book from huge collections of books on the Internet is very tedious work. In our system we are presenting a recommendation technique based on opinion mining to propose top ranked books on different domains. Based on the need of the customers and the reviews collected from them, we have categorized features for the books. We analyze the features on the basis of several characteristics that we have categorized and reviews of the users. . Weights are assigned to categorized features according to their importance and usage, and accordingly the ranks are given. Finally, top ten ranked books are displayed. This method is expected to be useful for millions of users who seeks for desired books.

There are several known and frequent used techniques to recommend products, amongst which opinion mining is one of the emerging and efficient method being used worldwide. Customers' reviews are the basis for opinion mining technique. Finding and summarizing the opinion from huge amount of reviews from the customers, is also very tedious for business.

Opinion mining is a very hot topic in the field of data mining. The main issue to consider is to find (a) product feature and (b) analysis comments, whether positive or negative, as described in. People generally use some pre-determined terms to interpret it as a positive or negative comments, like, better, good, nice, well written, highly recommend etc. are treated as positive terms and worst, time consuming, bad, not recommended, etc. are termed as negative comments.
Opinion retrieval is perceived as a two-step task, finding relevant documents and re-ranking these documents by opinion scores. The reviews are given by the human and it is very evident that to understand the review one should perceive it as human being. Finding the comments only is not sufficient. Sometimes things are different then what they seem to appear. Let us consider the following example:

"I highly recommend this book for those who want to waste their time and money."

Though the sentence above has terms like highly recommend and better but both terms are being used in a negative sense for some specific book, keeping only the positive and negative aspects of the terms and processing on these basis is not sufficient alone to extract opinion for a better conclusion.

\section{CONCEPT OF OPINION MINING}

Textual information in the world can be broadly classified into two main categories, facts and opinions. Facts are objective statements about entities and events in the world. Opinions are subjective statements that reflect people's sentiments or perceptions about the entities and events. Much of the existing research on text information processing has been focused on mining and retrieval of factual information, e.g., information retrieval, Web search, and many other text mining and natural language processing tasks. Small amount of work has been done on the processing of opinions until only recently. Yet, opinions are so important that whenever one needs to make a decision one wants to hear opinions of other people. This is not only true for individuals but also true for organizations.

One can write reviews of products at sites and express views on almost anything in Internet forums, discussion groups, and blogs, which are collectively called the user generated content. Now if one wants to buy a product, it is no longer 
necessary to ask anyone because there are lots of product reviews on the Web which give the opinions of the existing users of the product. For a company, it may no longer need to conduct surveys, to organize focused groups or to employ external consultants in order to find consumer opinions about its products and those of its competitors.

Finding opinion sources and monitoring them on the Web, however, can still be a formidable task because a large number of diverse sources exist on the Web and each source also contains a huge volume of information. In many cases, opinions are hidden in long forum posts and blogs. It is very difficult for a human reader to find relevant sources, extract pertinent sentences, read them, summarize them and organize them into usable forms. An automated opinion mining and recommendation system is thus needed.

So we can say that, opinion mining is a technique for collecting and categorizing information about the product to track views of the people.

\section{ALGORITHMS USED}

Algorithms used are:-

1) Commtrust Algorithm

2) Normalization Algorithm

\subsection{Commtrust Algorithm}

A fine-grained multi-dimensional trust evaluation model by mining e-commerce feedback comments is projected; it is called as Comment-based Multi-dimensional trust(CommTrust). Comprehensive trust profiles are computed for sellers using CommTrust. It includes dimension reputation scores and weights and overall trust scores by aggregating dimension scores of reputation. The first system which calculates fine-grained multidimensional trust profiles automatically by mining feedback comments is CommTrust.

\subsubsection{The Representation of Stanford Typed}

\section{Dependencies}

To have a simple description of the grammatical relationships in a sentence which could very easily be understood and effectively used by people without linguistic expertise who wanted to extract textual relations, The representation of the Stanford typed dependencies was deliberated. As explained in , the representation was not designed for the intention of parser evaluation; Researchers agree that with the widespread sentiment that dependency-based evaluation of parsers avoids many of the problems of the traditional Perceval measures. Also to the extent that the Stanford dependency representation is an efficient representation for the tasks envisioned. It is perhaps closer to an appropriate task based evaluation than some of the alternative dependency representations available.

\subsubsection{Computational Trust Evaluation}

In literature, the effective rating bias in the eBay reputation system is well documentation. As proposed in , to examine feedback comments to bring seller reputation scores down to a rational scale. There comments that do not demonstrate explicit positive ratings are deemed negative ratings on transactions.

Similar to that buyers and sellers are referred to as individuals in e-commerce applications. Peers and agents are terms always used to indicate the individuals in open systems in various applications in the trust evaluation literature. The comprehensive overview of trust model is provided in. Individual level trust models aims to compute the reliability of peers and assist buyers in their work of decision making. To regulate the behavior of peers, avoid fraudsters and ensure system security was the system level models aim.

\subsection{Normalization Algorithm}

In normalized algorithm, we rank the books based on weights assigned to them. We have list of features about the books with the maximum weight. Based on user reviews we assign weights to these features of the book.

Then using following formula, we evaluate final score of that book which is called as normalized score of the book.

$$
\text { Normalized Opinion score }(N S)=T / M \text {------ (1) }
$$

Where,

$T=$ Sum of total weights assigned to the book

$M=$ Sum of the maximum weights that can be assigned to each feature of the book.

\section{CONCLUSION}

We have proposed effective algorithms to compute dimension trust scores and dimension weights automatically via extracting aspect opinion expressions from feedback comments and clustering them into dimensions. Our approach demonstrates the novel application of combining natural language processing with opinion mining and summarization techniques in trust evaluation for e-commerce applications.

We intend to present a recommender technique to recommend top $\mathrm{N}$ products for some specific type, in this paper the product is book and $\mathrm{N}$ is 10 . We searched for top books on various topics and categorized the features; based upon these features we perform the ranking. The weights are given to several features and the weights are assigned according to the importance of the feature and requirement of the users.

Finally, top ten books are listed. These suggested ranked books are supposed to be one of the leading books on the topic concerned, and this work may helpful for millions of customers who seek for the best books available in the market. 


\section{REFERENCES}

[1]. IEEE(July'14)"Social Voting Advice Applications Definitions, Challenges, Datasets and Evaluation" by Ioannis Katakis, Nicolas Tsapatsoulis, Fernando Mendez, Vasiliki Triga, and Constantinos Djouvas.

[2]. IEEE(July'14)"CommTrust: Computing MultiDimensional Trust by Mining E-Commerce Feedback Comments" byXiuzhen Zhang, Lishan Cui, and Yan Wang, Senior Member, IEEE.

[3]. IEEE(June'13)“ Book Recommendation System Using Opinion Mining Technique" byShahab Saquib Sohail, Jamshed Siddiqui, Rashid Ali. 